outcomes, and interviewer-administered alcohol consumption questions, at age 26, 32 and 38 years.

Results Response level was $>90 \%$ at each assessment. At 38, drinking before or during sex in the previous year was common (8.2\% of men; $14.6 \%$ of women reported "usually/always"), and unwanted consequences were reported by $13.5 \%$ of men and $11.9 \%$ of women, including regretted sex or failure to use contraception or condoms. Frequent heavy drinkers were more likely to "use alcohol to make it easier to have sex" and regret partner choice, particularly women. Heavy drinking frequency was strongly associated with partner numbers for men and women at 32, but only for women at 38 . Significantly higher odds of STIs amongst the heaviest drinking men, and TOPs amongst the heaviest drinking women were seen at 32-38.

Conclusion Alcohol involvement in sex continues beyond young adulthood where it has been well documented, and is common at 38. Women appear to be more affected than men, and heavy drinking is associated with poorer outcomes for both. Improving sexual health and wellbeing throughout the life course needs to take account of the role of alcohol in sexual behaviour.

Disclosure of interest statement This work was supported by the Health Research Council of New Zealand [12/1086]. The authors have no conflicts of interest.

\section{P04.20 INCIDENT SEXUALLY TRANSMITTED INFECTION AND POST-INFECTION PARTNER CHANGE}

${ }^{1} \mathrm{JD}$ Fortenberry ${ }^{*},{ }^{2} \mathrm{~F} \mathrm{He},{ }^{2} \mathrm{~J}$ Harezlak, ${ }^{1} \mathrm{DJ}$ Hensel. ${ }^{1}$ Indiana University School of Medicine; ${ }^{2}$ Indiana University Fairbanks School of Public Health

\subsection{6/sextrans-2015-052270.274}

Introduction Sex partner change is necessary to maintain STI within a population. However, most people change partners relatively infrequently, and the timing of partner changes relative to incident STI is poorly understood.

Methods 272 participants without STI at enrollment (18-29 years of age; $126(46 \%)$ men/146 (54\%) women) from a high STI population completed a 12 -week study of daily reports of partner-specific sexual behaviours. None reported commercial sex work. Weekly self-obtained vaginal or urine samples were tested (and treated, if positive) at the end of 12-weeks for C trachomatis (CT), $N$ gonorrhoeae (GC), and T vaginalis (TV) using commercially available NAAT. Survival analysis techniques were used to describe time to first partner change following first $\mathrm{NAAT}+$.

Results 15, 12, and 23 participants acquired an incident CT+, GC+, or TV+ NAAT. Partner change after the first NAAT+ test was seen for $7(47 \%), 6(50 \%)$, and $3(13 \%)$ participants with $\mathrm{CT}+, \mathrm{GC}+$, or TV+ NAAT, respectively. Median time to partner change after first NAAT +7.6 and 4.6 weeks following $\mathrm{CT}+$ or GC+, respectively. Among 231 participants with no incident NAAT+ during 12 weeks of followup, 89 (39\%) changed partners.

Conclusion Persons with incident STI have higher rates of partner change than those without incident STI. Rapid partner change is common following a new CT or GC infection, but less common for TV. Interventions focused on short-term partner change could be especially effective approaches to CT and GC control efforts.

Disclosure of interest statement This research was funded by the National Institute of Child Health and Human Development. The authors have no relevant conflict of interest to disclose.

\section{P04.21 EVENT-LEVEL ANALYSIS OF CONDOM USE AND LOVE DURING TRANSACTIONAL VAGINAL INTERCOURSE AMONG WOMEN LIVING IN THE MIDWESTERN UNITED STATES}

${ }^{1} \mathrm{AM}$ Roth, ${ }^{2} \mathrm{KD}$ Wagner, ${ }^{3} \mathrm{DJ}$ Hensel, ${ }^{1} \mathrm{Q}$ Truong, ${ }^{3} \mathrm{SE}$ Wiehe, ${ }^{4} \mathrm{JG}$ Rosenberger, ${ }^{3} \mathrm{JD}$ Fortenberry*. 'Drexel University; ${ }^{2}$ University of Nevada, Reno; ${ }^{3}$ Indiana University; ${ }^{4}$ George Mason University

\subsection{6/sextrans-2015-052270.275}

Introduction Little research has explored how love affects condom use among women engaging in transactional sex. Most interventions for these women focus on non-romantic partners who may not confer the most sexual risk for HIV/STI.

Methods 26 women were enrolled into 4-week study during which event-level information about mood and sexual intercourse was collected twice daily via cell phone. We used descriptive statistics and binary logistic regression with generalised estimating equation correction to test associations between person and event-level factors and condom use during transactional sex events.

Results Participants reported 88 paid/traded vaginal intercourse events; $50.2 \%$ were condom protected. After controlling for partner type, feeling in love on a given day was significantly associated with higher odds of condom use, while time of day and day of were significantly associated with lower odds of condom use. There was a significant interaction between being in love and using condoms $(\mathrm{p}=0.03)$. When participants reported being in love, they used condoms during $57.1 \%$ of events with romantic partners and $61.1 \%$ of events with non-romantic partners. When women did not report being in love, they reported condom use in $64.3 \%$ of events with non-romantic partners and $54.0 \%$ of events with romantic partners.

Conclusion There was a strong association between being in love on a given day and condom use during transactional events. This association was moderated by partner type such that when women reported being in love they were more likely to use condoms with non-romantic partners and less likely to use condoms with romantic partners. Love may mark increased STI/HIV risk in romantic relationships, especially if condoms are seen as barriers to intimacy that distinguishes romantic from non-romantic partners. Unpacking women's partner-specific perceptions of risk may be help women make the link between affective states and preventive behaviours.

Disclosure of interest statement The study was funded by the Indiana University Purdue University Indianapolis Centre for Urban Health and NIDA grant T32 DA 023356.

\section{P04.22 SEX PARTNER MEETING PLACES OVER TIME AMONG NEWLY HIV DIAGNOSED MEN WHO HAVE SEX WITH MEN (MSM) IN BALTIMORE, MARYLAND}

${ }^{1,2} \mathrm{JM}$ Jennings, ${ }^{2} \mathrm{ML}$ Reilly, ${ }^{1,3}$ J Perin, ${ }^{1,4} \mathrm{C}$ Schumacher, ${ }^{1} \mathrm{M}$ Sharma, ${ }^{1} \mathrm{AG}$ Safi, ${ }^{1} \mathrm{EL}$ Fields, ${ }^{4} \mathrm{R}$ Murva, ${ }^{4} \mathrm{C}$ Nganga-Good, $4,5,6 \mathrm{P}$ Chaulk. ${ }^{1}$ Center for Child and Community Health Research, Department of Pediatrics, Johns Hopkins School of Medicine, Baltimore, MD, USA; ${ }^{2}$ Department of Epidemiology, John Hopkins Bloomberg School of Public Health, Baltimore, MD, USA; ${ }^{3}$ Department of International Health, John Hopkins Bloomberg School of Public Health, Baltimore, MD, USA; ${ }^{4}$ Baltimore City Health Department, Baltimore, MD, USA; ${ }^{5}$ Department of Health Policy and Management, John Hopkins Bloomberg School of Public Health, Baltimore, MD, USA; ${ }^{6}$ Johns Hopkins School of Medicine, Baltimore, MD, USA

10.1136/sextrans-2015-052270.276 
Introduction Evidence suggests that sex partner meeting places may be important locales to access men who have sex with men (MSM) to implement targeted HIV control strategies. Little is known about how patterns of these places have changed over time and whether there are new and emerging meeting places.

Methods The objectives of this study were to 1) describe the frequency of report of MSM sex partner meeting places over time, 2) to compare frequently reported meeting places over a five year period and in the past year, and 3) in a subset analysis, compare distributions of mean HIV viral loads, as a proxy for HIV transmission risk, of reported meeting places among newly HIV diagnosed MSM in Baltimore City, Maryland.

Results 869 sex partner meeting places were reported, including 306 unique places. Internet-based sites (38\%) and bars/clubs (31\%) were the most frequently reported meeting place typologies. Over the five year period there was variability in the frequencies of reports of meeting place typologies over time. Among internet-based sites and bars/clubs, 3/5 and 4/5 respectively most frequently reported sites over the five years were also in the top five most frequently reported in the most recent year. The distribution of mean viral load by place typology was not significantly different overall (Fisher's exact $p=0.700$ ) or between bars/clubs compared to internet-based sites (Fisher's exact $\mathrm{p}=0.863$ ).

Conclusion This study provides a richer understanding of sex partner meeting places reported by MSM over time and their transmission potential.

Disclosure of interest statement The Centre for Child and Community Health Research (CCHR) and the Baltimore City Health Department were funded for this work by the Centres for Disease Control and Prevention and the NIH. No pharmaceutical grants were received in the development of this study.

\section{P04.23 MIND THE GAP! BIOLOGICAL AND BEHAVIOURAL CONCURRENCY AND THEIR POTENTIAL FOR STI TRANSMISSION IN THE BRITISH POPULATION}

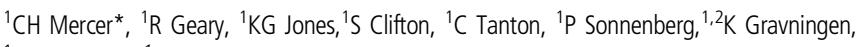
${ }^{1}$ AM Johnson. ${ }^{1}$ Centre for Sexual Health \& HIV Research, Research Department of Infection \& Population Health, University College London; ${ }^{2}$ Department of Microbiology and Infection
\end{abstract} Control, University Hospital of North Norway

\subsection{6/sextrans-2015-052270.277}

Background Key drivers for STI transmission are the number of sexual partners and their timing. Concurrent partnerships are a known risk factor but non-overlapping (serially monogamous) partnerships may be biologically concurrent for some STIs where infectious periods extend across the 'gap'. We examine recent partnership histories among people aged 16-44y in Britain and how they relate to reported STI diagnoses.

Methods Analyses of data collected for Britain's third National Survey of Sexual Attitudes and Lifestyles, a probability survey undertaken 2010-2012 with 9,902 people aged 16-44y. Computer-assisted self-interviews included questions about participants' 3 most recent partners and STI diagnosis history. Gaps between partners were calculated from the dates of most recent sex with a former partner and first sex with the next partner.

Results Over $90 \%$ of men and women reported $>=1$ partners in the past $5 \mathrm{y}$, of whom, $51.2 \%$ (men) and $42.5 \%$ (women) reported $>=2$ partners during this time and so had had $>=1$ gaps (total: 7, 724 gaps). The median gap was 2 months (IQR:-3 to 8 months) with two-thirds of all gaps $>=1$ month denoting serial monogamy. One-quarter (95\% CI: $23.8 \%-26.2 \%$ ) of all gaps were 0-3 months, while $21.8 \%$ (95\% CI: 20.7\%-22.9\%) were $>=12$ months. In multivariable analyses which adjusted for total partner numbers (past 5y), reported STI diagnoses (past $5 y$ ) were as common among participants whose recent partners had been concurrent as serially monogamous participants where the gap was $<=4$ months among men: $10.3 \%$ (95\% CI: 7.6\%-13.9\%) vs. $11.3 \%$ (95\% CI: $8.9 \%-14.3 \%)$, and $<=12$ months among women: $12.3 \%$ (95\% CI: 9.2\%-16.3\%) vs. 11.5\% (95\% CI: 9.7\%-13.6\%).

Conclusion While people in Britain have few partners on average and the majority with multiple partners are serially monogamous, the gap between partners is often shorter than the infectious period of common STIs and so effectively biologically concurrent. STI prevention efforts need to address this and should encourage STI testing prior to unprotected sex with new partners, even if serially monogamous.

Disclosure of interest statement AMJ has been a Governor of the Wellcome Trust since 2011. The other authors declare that they have no conflicts of interest.

\section{P04.24 EVALUATION OF PREVALENCE OF STIS BETWEEN WOMEN REFERRED TO "COUNSELLING AND HARM REDUCTION CENTRE FOR VULNERABLE WOMEN" IN ISFAHAN - IRAN 2014}

M Meshkati*, S Narimani, M Zandieh, R Fadai Nobari. Isfahan University of Medical Sceiences, Province Health Center

\subsection{6/sextrans-2015-052270.278}

Introduction At this time sexually transmitted infections are a major public health problem. Prevalence of STIs is correlated with distribution of sexual behaviours. Rates of sexual activity among Iranian youth were low but it is gradually changing. In the other hand rate of sexual transmission of HIV among newly identified people living with HIV is increasing year by year that might have probably a consistent trend with STIs. By this way Counselling and Harm Reduction centres for vulnerable women were established. So we looked clients in these centres for evaluating prevalence of STIs base on syndromic approach.

Methods In Isfahan, we lunched 2 centres for vulnerable women. We performed a survey (cross - sectional) for all clients who referred in 2014. The data was gathered by the client's files.

Results We have admitted 160 clients, the majority of them were in group ages $25-34$ years (38.4\%) and married (81\%). $47 \%$ of clients had history of sexual high risk behaviour that $50 \%$ were drug abuser. All of clients were assessed in every visit to centre for signs of STIs.30\% of them need to exam and 50\% of them were treated because vaginal discharge.

Conclusion These results show the increase of sexual high risk behaviours in young people and special between drug abuser. So it is necessary to evaluate STIs between this group. In this time, we have to manage base on syndromic approach so we need to provide etiologic approach for improving care and treatment. We have faced risk of third episode of HIV/AIDS from IDU to sexual contact in Iran right now so it is essential to improve harm reduction centres as well as social support in order to empower women for job finding that could play an effective and important role in control of spreading HIV/AIDS and STIs.

Disclosure of interest Counselling and Harm Reduction centres for vulnerable women were founded by Isfahan university of medical sciences. 